\title{
LISTA DE PROYECTOS DE GRADUACIÓN DE GRADO Y DE POSGRADO DEL AÑO 2008 DE LA FACULTAD DE INGENIERÍA DE LA UNIVERSIDAD DE COSTA RICA
}

ESCUELA DE ARQUITECTURA

\section{GRADO DE LICENCIATURA}

Aguilar Bonilla, Kenneth; Rodríguez Pérez, Erick. Estación intermodal en Desamparados.

Alvarado Rodríguez, Carlos Antonio. Centro Comunal Cascajal de Coronado.

Araya Jiménez, Alejandra; Marín Murillo, Alejandra. Sistema urbano de parqueo inteligente.

Calvo Ramírez, Yeimy. Centro Urbano de la Cultura y el Arte para el distrito Central del Cantón de Curridabat.

Chaverri Cerdas, Erika; Von Breymann Miranda, Helga. Nuevos territorios anfibios: planificación e intervención estratégica de la ciudad de Puntarenas ante el impacto del cambio climático.

Chaves González, José Francisco. Complejo deportivo especializado para atletismo de alto rendimiento Sanatorio Durán y propuesta de distribución territorial para un CAR.

Cordero Hidalgo, William. Clínica integral de terapia y rehabilitación.

Erfling Mayorga, Fanny. Centro de Investigación Ambiental Refugio Nacional de Vida Silvestre Ostional.

Espinoza Espinoza, Carlos. Aportes a la acústica y diseño de sala de conciertos para música lírica y orquestal.

Esquivel Suárez, Paola; Madrigal Madrigal, Ana Cristina. Centro de Convenciones Internacionales para el Área Metropolitana.

González Alvarado, Carolina. Diseño de los bordes urbanos y las franjas de protección del Río Ciruelas a través de la creación de un parque lineal.

González Bolaños, Juan Carlos. Centro Cívico de Corredores. 
González Sancho, Susana. Auditorio de la Universidad para la Paz.

Gutiérrez Flores, Marcela. Ú-SIWO "La casa del viento y el conocimiento de los Bribri - Cabécar".

Hernández Murillo, Eleonora. Albergue Los Cerros: propuesta de arquitectura bioclimática para turismo rural en Nicoya, Guanacaste.

Jiménez Navarro, Jaime; Young Leitón, Mario. Centro Folklórico de Tradiciones Limonenses.

Montoya Arroyo, Franklin. Estación intermodal de transporte público para la ciudad de Alajuela.

Murillo Ramírez, Marianela. Centro Agroecoturístico Comunitario Poasito de Sabanilla de Alajuela.

Porras Villareal, María del Pilar. Parque para la conservación de fauna silvestre: Zoo - Inmersión, Santa Ana.

Quesada Zúñiga, José David. Centro para el Desarrollo de la Artes de Pérez Zeledón en el marco de la propuesta conceptual: parque lineal Río San Isidro.

Sáenz Corella, Douglas E. Renovación urbana en el centro de Desamparados.

Salas Alfaro, Pamela. Centro Educativo Comunal para la Escuela Rogelio Fernández Güell, Ciudad Colón, Mora.

Santa María Ferdinand, Cristóbal; McRae Baldi, Alejandra. Espacio público y movilidad: ciclo ruta urbana para San José, circuito oeste distritos: Pavas - Sabana - Hospital.

Solano Meza, Natalia. Recorrido imaginario: un ejercicio en el desarrollo de pautas de intervención urbana desde la imaginación ciudadana.

Vargas Ortega, Lía Rebeca. Arquitectura penitenciaria: modelo para el desarrollo de un centro penitenciario en Nicoya.

Vilaplana Chacón, Alejandra. Capri, propuesta alternativa de vivienda y atención integral.

\section{GRADO DE MAESTRÍA EN ARQUITECTURA Y CONSTRUCCIÓN}

Bedoya Calderón, Luis. Estudio de factibilidad de proyecto turístico Puerto San Luis - Lago Arenal. 
Blanco Villalobos, Minor. Análisis y reformulación del Centro Vacacional de Arenal hotel cinco estrellas.

Carmiol Barboza, Catalina. Reformulación del proyecto "Desarrollo Turístico Canales de Tortuguero".

Masís Esquivel, Alfonso. Condominio Residencial 14 Monos.

\section{GRADO DE MAESTRÍA PROFESIONAL EN VIVIENDA Y EQUIPAMIENTO SOCIAL}

Kauffmann Incer, Catherine. Diseño de sitio para la propuesta de consolidación en sitio de la comunidad Gracias a Dios.

Liberoff Nemirovsky, Jenny. Metodología para la intervención de asentamientos informales: un enfoque integral.

Saborío Morales, Rebeca. Espacios públicos, comunidad y participación: algunos conceptos a considerar en un proyecto de erradicación y reconstrucción de asentamientos en condición de precario y de tugurio.

Tioli Porras, Giovanna María. t Una propuesta de vivienda: asentamiento informal Gracias a Dios.

\section{GRADO DE MAESTRÍA EN PAISAJISMO Y DISEÑO DE SITIO}

Chaverri Flores, Laura. Parque articulador biológico Río Torres.

López Valverde, Óscar. Ciudad de Cartago movilidad, paisajes y símbolo.

Soto Bernardini, Laura. Diseño de un modelo turístico sostenible para el desarrollo y la conservación de una zona de protección.

Ureña Gómez, José Fabio. Conectores paisajísticos urbanos entre la Sede Rodrigo Facio de la Universidad de Costa Rica y el casco central del cantón de Montes de Oca.

Zúñiga León, Roxana. Propuesta de renovación paisajística para el Liceo José Joaquín Vargas Calvo.

\section{GRADO DE MAESTRÍA EN DISEÑO URBANO}

Vargas Guillén, Jaime. Centro dinamizador de actividades para Rincón Grande de Pavas. 


\section{ESCUELA DE INGENIERÍA AGRÍCOLA}

\section{GRADO DE LICENCIATURA}

Monge Jeremías, Roberto. Análisis de requerimientos, diseño y construcción de un simulador de lluvia portátil, para la cuantificación de la infiltración, escorrentía y erosión.

Prado Segura, Rodolfo. Metodología ajustada para la determinación de pérdidas de suelo.

\section{ESCUELA DE INGENIERÍA CIVIL}

\section{GRADO DE LICENCIATURA}

Agüero Porras, José Gerardo. Metodología de reciclaje de desechos electrónicos.

Alfaro Alpízar, Alexander. Evaluación y análisis comparativo de los métodos de estimación de costos de mano de obra.

Álvarez Barrantes, Lucrecia. Modelo hidrodinámico del embalse de almacenamiento de la Planta Hidroeléctrica Aguas Zarcas.

Barrantes Vargas, Hernán Guillelmo. Análisis de la razón de períodos de oscilación rotacional/trasnacional con la razón de rigideces torsional/lateral del piso representativo.

Benavides Aguilar, Carlos. Estudio de herramientas aplicadas a la construcción.

Castro Morales, Edward Jesús. Propuesta de modificación al reglamento a la ley 7600 de Costa Rica.

Cerdas Campos, Christian Alberto. Administración de contratos de construcción.

Cruz White, Armando. Metodología para la determinación de los rendimientos de las actividades constructivas de una línea de distribución eléctrica. Aplicado al ejemplo práctico de la obra L. D. Chacarita-Palmar Norte del Centro de Servicio de Construcción de la UEN PYSA, ICE.

Chavarría Madrigal, Cinthia. Propuesta constructiva para reducir el riesgo de incendio y mejorar las condiciones de seguridad humana en la Facultad de Ingeniería.

Fonseca Bolaños, Mauricio. Evaluación del riesgo por incendio de la Escuela de Arquitectura de la Universidad de Costa Rica. 
Fonseca Quesada, María José. Criterios para la elaboración de un sistema de administración de puentes a nivel nacional.

Gatjens Mora, Dennis. Desarrollo de herramientas para el control de avance y costos en proyectos de generación eléctrica: el proyecto hidroeléctrico Toro 3 del ICE.

González Mora, Esteban. Revisión del costo de obra gris y acabados en viviendas de interés social en Costa Rica.

Hernández Arguedas, Marco Antonio. Evaluación cuantitativa del recurso hídrico y gestión de manejo integral de la subcuenca Santa Lucía, Abangares.

Jiménez Elizondo, Joseph. Aplicación del método comparativo de mercado del sector inmobiliario.

Lizano Van Der Laat, Federico. Análisis cuantitativo de los compuestos orgánicos volátiles en el sector de construcción.

Meza Salas, Roberto Andrés. Implementación del sistema de evaluación LEED-NC Y.2.2 en empresas consultoras de ingeniería y arquitectura en Costa Rica.

Mora Solano, Leonardo. Opciones de ahorro energético y de recursos en el diseño de la vivienda unifamiliar.

Naranjo Castillo, Vladimir. Respuesta de paredes de vivienda ante cargas de impacto.

Orozco Campos, Eddy Gerardo. Alternativas constructivas de solución a riesgos por incendio en la Escuela de Química de la Universidad de Costa Rica.

Reyes Bonilla, Andrés. Estudio económico y recomendaciones de buenas prácticas constructivas de opciones de entrepiso para viviendas.

Rodríguez Arce, Juan Carlos. Diseño de taludes en roca para la contrapresa P. H. Pirrís.

Rodríguez Rojas, Greivin. Métodos físico-químicos para la eliminación de ácidos húmicos en fuentes de agua para consumo humano.

Roldán AbeIlán, Ariel Ricardo. Propuesta de una metodología para utilizar el método de los coeficientes de desplazamiento de FEMA-440 con el Código Sísmico de Costa Rica 2002.

Salas Pérez, Melissa. Análisis integral de optimización del acueducto de Lagunillas de Garabito Puntarenas.

Sánchez Campos, Fernando Alberto. Concretos durables con resistencia a la penetración de cloruros para estructuras en zonas costeras o con influencia del ambiente marino en la región Pacífico Central. 
Solórzano Quirós, Yadiana. Evaluación de un humedal como sistema complementario de tratamiento y drenaje para suelos con baja capacidad de infiltración.

Toruño Barrantes, Melvin Yanán. Propuesta de alternativas constructivas para disminuir el riesgo por incendio de los edificios de la Escuela de Ciencias de la Computación e Informática y del Centro de Informática de la Universidad de Costa Rica.

Vargas Torres, Ronny. Metodología de la Ingeniería del Valor aplicada a la construcción de viviendas unifamiliares en serie.

Villalobos Slon, Hernán. Calibración del modelo matemático QUAL2K para el análisis de calidad del agua para el caso de un río específico en Costa Rica.

\section{ESCUELA DE INGENIERÍA ELÉCTRICA}

\section{GRADO DE BACHILLERATO}

Alvarado Sáenz, Esteban José. Diseño y control de un vehículo no tripulado guiado por GPS.

Amado Martínez, Félix Fernando. Técnicas de acceso múltiple y algoritmos de detección de múltiples usuarios en telefonía celular: análisis comparativo.

Amado Martínez, María José. Verificación de funcionamiento del sistema de mantenimiento a bordo para el altímetro de radio digital de bajo rango (Digital Low Range Radio Altimeter Onboard Maintenance System DLRA-OMS)

Arias Alpízar, Cristiano Evaluación del impacto de la implementación del manual de redes de distribución eléctrica subterránea 19,9/34,5 kY.

Arias Avendaño, Fabio Andrés. Desarrollo de procedimientos para espectrómetro marca CVI para captura de espectros de radiación óptica coherente e incoherente para LAFfA.

Arroyo Solórzano, David. Desarrollo y montaje del procedimiento para medición de la disfracción usando el programa Data Studio, para el LAFTLA.

Barquero Carranza, Ricardo. Desarrollo de un modelo sintetizable para la CPUCR de 32 bits con pipeline.

Barrantes Dall' Anese, José Francisco. Programa para la identificación de procesos y la sintonización de controladores PID de dos grados de libertad.

Benavides Vargas, Andrea. Propuesta de implementación de un sistema para automatizar la administración de la red del Banco Nacional de Costa Rica. 
Bolaños Rodríguez, Gary. Procedimiento para mejora del uso del espectrofotómetro marca PASCO usando el programa Data Studio aplicado a estudios radiométricos para el LAFTLA.

Bolaños Vásquez, Jeremy. Especificación e implementación de plantas de emergencia para baja tensión.

Bonilla Trejos, Alberto. Costo de la energía no servida en Costa Rica.

Castro Rojas, Adrián Alberto. Sensores utilizados en la automatización industrial.

Cerdas Zeledón, Allan. Guía para el diseño de sistemas de puesta a tierra en instalaciones de telecomunicaciones de más de un nivel, aplicado en el edificio de telecomunicación del ICE en San Pedro de Montes de Oca.

Chacón Quesada, Rodrigo. Digitalizador de señales de banda base compleja utilizando el TDK320VC5416.

Corrales Acuña, Allan Arturo. Análisis de la tecnología HDTV y articulación de una propuesta para su implementación en el país.

Donadio Dinis, Roberto. Guía básica de protección contra incendios con énfasis en sistemas de alarma y detección de incendios en conformidad con el estándar NFPA 72.

Espinoza González, Martín. Análisis de tráfico en sistemas inalámbricos.

Fonseca Hernández, Jorge. Procedimiento de captura de imagen y vídeo, implementando un microscopio digital con cámara CCD SONY, para el análisis de fibras ópticas en el LAFTLA.

Guillén Torelli, Mauricio Alonso. Desarrollo de un modelo sintetizable para la CPUCR de 32 bits con pipeline.

Gutiérrez Rivera, Fernando. Demodulador de señales PAM digitalizadas utilizando el TDK320VC54 16.

Mora Zúñiga, Rafael. Programa estimador de mano de obra de cableado estructurado de edificios.

Morales Chacón, Óscar Andrés. Implementación de un codificador y decodificador fuente para un sistema de comunicación digital que utilice el algoritmo de codificación aritmética.

Navarro Retana, Rodolfo. Diseño de un transmisor de velocidad para el conjunto motor generador.

Paniagua, Luis Carlos. Criterios de diseño de antenas inteligentes. 
Pérez Arroyo, Heiner Alfredo. Magnificación de sobretensiones por maniobras de bancos de condensadores

Porras Huete, Carlos Roberto. Energía renovable: estudio de sistemas combinados de 200W-H/día-20000W-H/día.

Prado Mora, Carlos Roberto. Sistema eléctrico fotovoltáico para comunidad aislada.

Quirós Ramos, Diego Geovanny. Desarrollo de un procedimiento de pruebas de aceptación en fábrica (FAT, por sus siglas en inglés) para tableros de control y protección en subestaciones de mediana y alta tensión.

Quirós Tortós, Jairo. Puesta en marcha de la protección de impedancia ABB REL 670.

Ramírez Gutiérrez, Esteban. Mejores prácticas en el diseño de sistemas de alimentación eléctrica con aplicación a sistemas de control de invernaderos utilizando energía solar.

Ramos Fonseca, Pedro. Elaboración de un manual para redes de distribución eléctrica subterránea para $24,9 \mathrm{kY}$.

Rodríguez Retana, Jason. Pruebas para el cálculo del circuito equivalente y de la eficiencia en transformadores de distribución.

Ruiz Gutiérrez, Manfred. Equivalente computacional de un turbogenerador sincrónico de la empresa CATSA.

Santamaría, Julio. Programa estimador de mano de obra-zonas peligrosas.

Solís Carballo, Emmanuel Eugenio. El espectro radioeléctrico en Costa Rica.

Sterling Aguilar, Carlos. Propuesta para el diseño del sistema de distribución de energía eléctrica de la sala de gestión de banda ancha del edificio de telecomunicaciones del ICE en San Pedro de Montes de Oca.

Torres Arroyo, Elías Gerardo. Conteo remoto de asistentes a la Feria Vocacional de la Universidad de Costa Rica.

Villalobos Villalobos, Mario. Diseño de una interfaz de potencia para el conjunto motor generador del laboratorio de automática.

\section{GRADO DE LICENCIATURA}

Andrés Jácome, Luis Fernando. Análisis y proyección de la demanda eléctrica de la CNFL para la planificación de obras a mediano plazo, utilizando la tecnología del SIG. 
Castro Jiménez, Gerardo. Procedimiento de diseño e implementación de circuitos digitales utilizando herramientas EDA de código abierto.

Garro Calvo, MichaeI. Equivalente dinámico de granjas eólicas para estudios de estabilidad transitoria.

Méndez Arias, Verny. Desempeño y robustez de los lazos de control PID.

\section{MAESTRÍA ACADÉMICA EN INGENIERÍA ELÉCTRICA CON ÉNFASIS EN SISTEMAS DIGITALES}

Siles Canales, Francisco. Multianálisis de componentes principales en el dominio wavelet para detección de caras.

\section{MAESTRÍA PROFESIONAL EN SISTEMAS DE BAJA Y MEDIANA TENSIÓN}

Fernández Rodríguez, David. Sistemas eléctricos de una planta de tratamiento de aguas residuales.

\section{MAESTRÍA PROFESIONAL EN INGENIERÍA ELÉCTRICA, ÉNFASIS} EN COMUNICACIONES DIGITALES

Pereira Fuentes, Nelson. Análisis de calidad de serVICIO en la red avanzada de INTERNET del Instituto Costarricense de Electricidad.

Mora Castro, José Eliécer. Diagnóstico y mantenimiento del enlace por fibra óptica de los edificios de la Facultad de Ingeniería de la Universidad de Costa Rica.

Miranda Izquierdo, Rodrigo. Vídeo multicast sobre la red MPLS de la Universidad de Costa Rica.

Murillo Marín, Melvin Cristiano Determinación de las zonas de cobertura Proyecto Servicios Móviles Avanzados 3G.

\section{ESCUELA DE INGENIERÍA INDUSTRIAL}

\section{GRADO DE LICENCIATURA}

Alfaro González, Carolina; Durán, Ana Cristina; Jaikel, Kemly. Desarrollo participativo del marco y plan estratégico para una empresa de servicios eléctricos y telecomunicaciones. 
Alfaro Loría, Carolina; Camacho Lestón, Martiza; Montero Cascante, Mariano. Propuesta de un sistema de información para el mejoramiento de las operaciones de distribución de alimentos.

Barrantes Molina, Mauricio; Prado Villena, Guido. Reforma del estado gestión de operaciones y manufactura.

Cartín, José; L1obet, Pablo. Propuesta básica de una ontología informática de técnicas de muestreo estadístico.

De Mezerville Picado, André; Moser Meza, Stefan. Gestión de operaciones, manufactura y gestión de calidad.

Esquivel Granados, Ana Catalina; Ramos Hernández, Leinar. Gestión del manejo de recursos logísticos.

Forero Mercado, Alexandra; Montes de Oca Miranda, Sergio; Valenciano Gutiérrez, Michael. Certificación de productos.

Grau Vargas, Francisco; Soto Fournier, Paula; Bontempo García, Giannina. Propuesta de un modelo de manejo de desechos inorgánicos recuperables para la zona de Santa Elena.

Hernández González, Emelina; Cazanga Soto, Javier. Gestión de información.

Herrera Masís, Carlos; Rodríguez Morice, Mauricio; Andrade Garnier, Eduardo. Diseño de un sistema logístico de almacenamiento y administración de inventarios.

Leitón Díaz, Pablo; Vargas Benavides, A. Catalina. Gestión de operaciones y manufactura.

Odio Pozuelo, Tomás; Di Palma Van der Laat, Marco; Soto Quijano, Adriana. Rediseño del proceso de distribución de DHL.

Retana Mora, Flory; Barley Zúñiga, Sherbie; Ortega Cubero, Diana. Modelo de control estratégico para el área de tecnología de información.

Sánchez Rojas, Mauricio; Villar Blanco, Karla. Gestión de calidad.

Seco Padilla, José Daniel; Arauz Sanabria, Johnny. Gestión de operaciones y manufactura.

Solano Alvarado, Juan Pablo; Burgos Bonilla, Manuel; Di Palma, Paola. Modelo de gestión de operaciones logísticas de almacenamiento.

Solís Jara, Jorge; Mata Castillo, RandaIl; Arce Hidalgo, Nelson. Diseño de un modelo para mejorar la gestión de la logística de almacenamiento del centro de distribución. 
Trejos Vásquez, José Rafael; Matarrita Guillén, Andrea; Soto Trujillo, Daniel. Propuesta de un modelo de evaluación de proyectos y su impacto en el desarrollo sostenible.

Vargas López, Armando; Gamboa Rodríguez, Carlos. Gestión de operaciones y manufactura.

\section{ESCUELA DE INGENIERÍA MECÁNICA}

\section{GRADO DE MAESTRÍA CON ÉNFASIS EN ENERGÍA}

Arias Pérez, Alfonso. Análisis del ruido y propuestas de medidas de mitigación para descargas atmosféricas de las previstas del campo geotérmico Miravalles.

\section{GRADO DE MAESTRÍA SISTEMAS DE MANUFACTURA Y MATERIALES}

Cubero Sesín, Mauricio. Optimización del proceso de impresión de flux sobre sustratos en el ensamble de microprocesadores.

Pirola Aguilar, Bernardo. Estudio y normativa sobre ambientes explosivos debido a polvos en la industria de proceso de madera.

Sánchez Calvo, Alejandra. Diseño de un mecanismo de enrollado de queso palmito para la Cooperativa de Productores de Leche, Dos Pinos, R. L.

\section{ESCUELA DE INGENIERÍA QUÍMICA}

\section{GRADO DE LICENCIATURA}

Arias Guerrero, Randall. Elaboración de una puesta de reglamento de vertidos de aguas residuales en el alcantarillado municipal de Alajuela.

Artavia Vega, Nelson. Diseño, construcción y puesta en marcha de un equipo de electrodeposición de cromo para el Laboratorio de Ingeniería Química.

Berzosa Jiménez, Martín. Evaluación del consumo de potencia en tanques agitados para sistemas no newtonianos en fase acuosa.

Calderón Castro, Jenny. Producción de hidroxiapatita a nivel de planta piloto a partir de huesos de ganado vacuno.

Caravaca Villegas, Silesky. Prefactibilidad técnica y costeo para la extracción de cafina utilizando dióxido de carbono en estado supercrítico. 
Cerdas Víquez, Milena. Caracterización del efecto del tratamiento térmico y electroquímico de la hidroxiapatita para aplicaciones médicas.

Chaves Di Luca, Federico. Simulación de los principales procesos de separación en la refinación de petróleo de una refinería simple.

Chaves Sánchez, María Gabriela. Auditoría energética en los serVICIOSde agua caliente y vapor en el área de vulcanización de una fábrica productora de llantas.

Chinchilla Saborío, Carlos. Producción de biodiesel a partir de cuatro variedades de palma aceitera.

Cordero Ortiz, Jorge. Comparación técnica y de costos para un proceso de microfiltrado y los diferentes procesos de tratamiento de aguas residuales domésticas utilizadas en Costa Rica.

Corella Quirós, Pedro Guillermo. Establecimiento de medidas para minimizar el consumo de agua en una planta de fabricación de llantas.

Corrales Ureña, Yendry Regina. Determinación del efecto de la temperatura y la presión en la producción de biodiesel mediante la transesterificacion del aceite de palma empleando óxido de magnesio como catalizador.

Di Stefano Zúñiga, Eric José. Caracterización de la zeolita producida en un laboratorio utilizando los lodos residuales y baños de decapado alcalino de una empresa dedicada a la manufactura de aluminio anodinado como fuente de alúmina.

Garro Mena, Leonardo. Desarrollo de un software para el cálculo de la letalidad en procesos de esterilización de alimentos.

González Jiménez, Vivian. Dimensionamiento de un sistema para el tratamiento de contaminantes atmosféricos de los hornos de tipo indirecto de un beneficio de café.

Le Roy Fernández, Hugo. Factibilidad técnica económica de una planta para la producción de metil y propil parabeno.

López Martines, Juan Pablo. Auditoría energética en las líneas de producción de baterías AA, C, D en una planta productora de pilas.

Miranda Morales, Bárbara Cristina. Evaluación del tiempo de sacarificación del almidón e inhibición de las enzimas por presencia de etanol durante la fermentación con S. cerevisae.

Mora Vargas, Daniela. Diseño de un sistema de almacenamiento de químicos para una industria procesadora de leche.

Mora Vega, Marilyn. Estudio de prefactibilidad para la implementación de un centro de acopio de desechos sólidos en el Gran Área Metropolitana. 
Ramírez Varas, Lautaro. Comparación del método de calibración ortogonal con otros métodos de subdivisión de la incertidumbre por el método de simulación numérica mediante Monte CarIo.

Rodríguez Bogantes, Ivana. Análisis técnico económico preliminar de una planta para la producción de jarabe de glucosa y fructosa vía enzimática a partir de zagú.

Rojas Fallas, Daniel. Prefactibilidad técnica y económica para la adición de un sistema de congelación de ñame pelado y troceado en una planta exportadora de tubérculos a partir de materia prima no apta para la exportación como producto fresco.

Rojas Paniagua, Hugo. Propuesta de un sistema de tratamiento terclanó para la reutilización de eficiente de una planta de tratamiento de aguas residuales existente.

Román Cabezas, Adriana. Evaluación del proceso de lixiviación en la separación de metales en desechos de vidrio de tubos de rayos catódicos.

Ruphuy Chan, Gabriela Patricia. Obtención y caracterización del producto de interesterificación de aceite de maíz con ácido acético.

Sandí Campos, Adrián. Establecimiento de criterios técnicos para el desarrollo de una propuesta de norma nacional para el manejo seguro de los desechos de las baterías que produzcan mayor impacto en el ambiente costarricense.

Solano Argüello, RoCÍo. Producción de hidroxiapatita a partir de huesos de ganado vacuno utilizando dos métodos de reprecipitación: la oxidación de un agente complejante y la aplicación de calor.

Solano Sánchez, Silvia Elena. Evaluación del efecto de las características de la fruta y de las condiciones de almacenamiento sobre la vida útil de trozos de dos variedades de piña.

Ulloa Echandi, Juliana. Mapeo de riesgos de una pequeña industria fabricante de tortillas de maíz.

Valladares Sobrado, Mariela. Evaluación del A 1203 hidratado proveniente de los lodos residuales de EXTRALUM S.A. como potenciales entendedores para la producción de pinturas arquitectónicas

Zumbado Salas, Shirley María. Elaboración de un plan de gestión ambiental para un laboratorio de disoluciones parenterales.

Zúñiga Riveras, Alberto Jesús. Factibilidad técnica y estimación de la inversión total de capital de un relleno de seguridad para la disposición de los residuos peligrosos generados en la Sede Central de la Universidad de Costa Rica. 
GRADO DE MAESTRÍA PROFESIONAL CON ÉNFASIS EN INGENIERÍA AMBIENTAL

Mora Gómez, Bernardo. Valoración de la calidad de los sistemas de tratamiento de desechos líquidos en Costa Rica. 